\title{
Influence of sodium nitroprusside on human erythrocyte membrane water permeability: an NMR study
}

\author{
Gojmir Lahajnar ${ }^{1}$, Barbara Sobotič ${ }^{1}$, Ana Sepe ${ }^{1}$, Vojko Jazbinšek ${ }^{2}$ and Slavko Pečar ${ }^{1,3}$ \\ ${ }^{1}$ Jožef Stefan Institute, Jamova 39, Ljubljana, Slovenia \\ ${ }^{2}$ Institute of Mathematics, Physics and Mechanics, Jadranska 19, Ljubljana, Slovenia \\ ${ }^{3}$ University of Ljubljana, Faculty of Pharmacy, Aškerčeva 7, Ljubljana, Slovenia
}

\begin{abstract}
Sodium nitroprusside (SNP) is a nitric oxide ( $\left.{ }^{\circ} \mathrm{NO}\right)$ donor in vitro and in vivo. In this paper the time variation of the intracellular water proton nuclear magnetic resonance (NMR) effective relaxation time $T_{2 \mathrm{a}}{ }_{2 \mathrm{a}}$ in SNP-treated human erythrocyte suspensions, containing $10 \mathrm{mM}$ membrane impermeable paramagnetic $\mathrm{MnCl}_{2}$, has been measured. The observed $T_{2 \mathrm{a}}$ time-course was analyzed in terms of the two mechanisms by which released ${ }^{\circ} \mathrm{NO}$ affects $T_{2 \mathrm{a}}$. These are, respectively, enhancement of the intracellular water proton intrinsic $\mathrm{NMR}$ relaxation rate $1 / T_{2 \mathrm{a}}$ by paramagnetism of ${ }^{\circ} \mathrm{NO}$ subsequently bonded to iron atoms of intracellular deoxyhemoglobin, and suppression of diffusional water permeability $P_{\mathrm{d}}$ as a consequence of nitrosylation of aquaporin-1 (AQP1) channel Cys189, either by direct reaction with ${ }^{\circ} \mathrm{NO}$ or with one of the ${ }^{\circ} \mathrm{NO}$ oxidation products, such as $\mathrm{N}_{2} \mathrm{O}_{3}$. The bound ${ }^{\circ} \mathrm{NO}$ on the Cys 189 thiol residue appears to impose a less efficient barrier to water permeation through AQP1 than the larger carboxyphenylmercuryl residue from $p$-chloromercuribenzoate. The effect of ${ }^{*} \mathrm{NO}$ on $P_{\mathrm{d}}$ is discussed in terms of NO-induced vasodilation.
\end{abstract}

Key words: Human erythrocytes - Water permeability coefficient — Sodium nitroprusside — Proton NMR transverse relaxation time

\section{Introduction}

Nitric oxide $\left({ }^{\circ} \mathrm{NO}\right)$ is a biologically important free radical, involved in many physiological and pathological processes (Ignarro 2000a). Unlike other radicals, it is relatively stable, reacting very fast only with other radical species. Although its role in regulating biological processes is well documented, the mechanism by which its unique chemical properties, fast and complex radical reactions account for its function in living matter, is not completely understood. It is known that ${ }^{\circ} \mathrm{NO}$ plays a unique role as a signalling molecule in the vascular system (Ignarro 2000b), where it binds on the heme iron ion of guanylate cyclase (Eich et al. 1996; Liao et al. 1999; Huang et al. 2001; Huang et al. 2002; Herold and Röck 2005), and that it functions as a neurotransmitter (Snyder and Bredt 1992). Due to its lipophilic character, ${ }^{*} \mathrm{NO}$, like oxygen, is accumulated in lipid membranes (Möller et al. 2007), where reactions between ${ }^{\circ} \mathrm{NO}$, oxygen and their products take

Correspondence to: Gojmir Lahajnar, J. Stefan Institute, Jamova 39, SI-1000 Ljubljana, Slovenia

E-mail: gojmir.lahajnar@ fmf.uni-lj.si place. As the lipid bilayer is a functional surrounding for many membrane-bound enzymes, transmembrane receptors and channels, the presence of ${ }^{\bullet} \mathrm{NO}$ and its products of oxidation can modulate the activity of membrane proteins. $\mathrm{N}_{2} \mathrm{O}_{3}$, which results from ${ }^{\circ} \mathrm{NO}$ oxidation in the membrane (Möller et al. 2007), is able to nitrosylate thiol groups of proteins (Shaw and Vosper 1977; Subczynski and Hyde 1983; Ye et al. 2001), which can be physiologically or pathologically important for posttranslational peptide modification leading to modified protein function.

We report the partial temporary inhibition of the diffusional water permeability $\left(P_{\mathrm{d}}\right)$ of the human erythrocyte membrane after treatment of erythrocyte suspension with sodium nitroprusside $\mathrm{Na}_{2}\left[\mathrm{Fe}(\mathrm{CN})_{5} \mathrm{NO}\right](\mathrm{SNP})$. The human erythrocyte membrane was the first plasma membrane recognized to contain many copies (some 200.000 per erythrocyte) of the specialized, water-specific, transmembrane channel protein (Denker et al. 1988; Preston and Agre 1991; Preston et al. 1992), now called aquaporin-1 (AQP1). The AQP1 channels allow large osmotically driven transmembrane water fluxes in certain cell species (including mammalian erythrocytes) that cannot be accounted for by lipid-mediated 
transmembrane water permeability alone. It is well known that channel-mediated transmembrane water passage in erythrocytes can be inhibited by organic mercurials (Macey and Farmer 1970), and this effect was subsequently shown to result from their reaction with the critical thiol $(-\mathrm{SH})$ group of cysteine 189 (Cys189) located near the constriction of the "hour-glass" AQP1 water channel (Preston et al. 1993). In the previous study (Lahajnar et al. 2007) it was suggested that treatment of erythrocytes with SNP causes partial reduction of membrane $P_{\mathrm{d}}$ as a result of ${ }^{\bullet} \mathrm{NO}$-induced formation, either directly or via its oxidation intermediate $\mathrm{N}_{2} \mathrm{O}_{3}$, of Snitrosothiols on Cys189. In the present study the supposed release of ${ }^{\bullet} \mathrm{NO}$ from $\mathrm{SNP}$ added to the suspension of human erythrocytes, and its suppressive influence on erythrocyte membrane $P_{\mathrm{d}}$, were studied in detail. The relevant information was deduced from induced variation in the measured proton $\left({ }^{1} \mathrm{H}\right)$ nuclear magnetic resonance (NMR) transverse relaxation $\left(T_{2}\right)$ function $M(t)$ of water exchanging between the intracellular and paramagnetic extracellular compartments of the erythrocyte suspension, the latter being doped with membrane-impermeable paramagnetic $\mathrm{Mn}^{2+}$ ions. This so called ${ }^{1} \mathrm{H}-\mathrm{T}_{2} \mathrm{NMR}$ method allows determination of the time constant $\tau_{\text {exch }}$ for transmembrane exchange of intracellular water, and as $P_{\mathrm{d}}$ is inversely proportional to $\tau_{\text {exch }}$, this method enables detection of any induced modification of erythrocyte membrane water permeability $P_{\mathrm{d}}$. The results appear to indicate a partial temporary restriction of water diffusion through the AQP1 channels (i.e., suppression of $P_{\mathrm{d}}$ ), suggested to be the consequence of S-nitrosylation of their Cys189. Comparative treatment of erythrocytes with $1 \mathrm{mM}$ p-chloromercuribenzoate (PCMB) was found to induce complete inhibition of the AQP1-mediated water permeation across the erythrocyte membrane.

\section{Materials and Methods}

\section{Sample preparation}

Erythrocyte samples were prepared from blood obtained by venipuncture from healthy male volunteers on the day of the experiments. Fresh citrated blood was washed three times with isotonic TRIS buffer $(\mathrm{pH}=7.4)$ by centrifugation $(5 \mathrm{~min}, 1500 \times g)$ at room temperature and twice with 330 mOsm Tris buffer $(\mathrm{pH}=7.4)$. A 40\% stock suspension of erythrocytes was prepared. The final concentration of erythrocytes in the samples of $\sim 0.3 \mathrm{ml}$ volume for NMR measurements was $\sim 20 \%$, and the final concentration of paramagnetic manganese ions in the form of $\mathrm{MnCl}_{2} 10 \mathrm{mM}$. SNP was added at $1,0.25$ and $0.1 \mathrm{mM}$ final concentrations in the cell suspensions. To have a reference to SNP-induced effects on membrane $P_{\mathrm{d}}$, erythrocytes were also treated with $1 \mathrm{mM}$ PCMB that fully inhibited the erythrocyte membrane
AQP1 channel water permeation. All measurements were performed on erythrocytes under isotonic conditions.

\section{NMR measurements and data analysis}

As discussed later, the ${ }^{1} \mathrm{H}-\mathrm{T}_{2} \mathrm{NMR}$ method enabled observation of a fast release of ${ }^{\bullet} \mathrm{NO}$ from SNP upon its addition to the erythrocyte suspension as well as provided information about PCMB- and SNP-induced reduction of the erythrocyte membrane diffusional water permeability $P_{\mathrm{d}}$. The ${ }^{1} \mathrm{H}-T_{2}$ NMR technique (Hazlewood et al. 1974; Pirkle et al. 1979; Lahajnar 1992) is based on measurements of the ${ }^{1} \mathrm{H}$ NMR transverse relaxation $\left(T_{2}\right)$ functions $M(t)$ of water exchanging between intracellular and paramagnetic extracellular compartments of the erythrocyte suspension, where the extracellular compartment is made paramagnetic by adding membrane-impermeable paramagnetic $\mathrm{Mn}^{2+}$. In this way, two essentially different NMR relaxation compartments of water protons in the cell suspension are created: the intracellular water (site a) and the $\mathrm{Mn}^{2+}$-doped paramagnetic extracellular solution (site b). However, the corresponding intrinsic proton NMR transverse or spinspin relaxation times $T_{2 \mathrm{a}}$ and $T_{2 \mathrm{~b}}$ are effectively modified to $T^{\prime}{ }_{2 \mathrm{a}}$ and $T^{\prime}{ }_{2 \mathrm{~b}}$ values, due to the finite mean life times $\tau_{\mathrm{a}}$ and $\tau_{\mathrm{b}}$ of water molecules in the sites (a) and (b) as a result of equilibrium diffusional exchange of water molecules between the two sites under the iso-osmotic conditions of the erythrocyte suspension. In this case, the proton NMR transverse or spin-spin magnetization relaxation behaviour is described by the set of coupled rate equations (Hazlewood et al. 1974)

$$
\begin{aligned}
& \mathrm{d}\left(\Delta m_{\mathrm{a}}\right) / \mathrm{d} t=-\left(\Delta m_{\mathrm{a}} / T_{2 \mathrm{a}}\right)-\left(\Delta m_{\mathrm{a}} / \tau_{\mathrm{a}}\right)+\Delta m_{\mathrm{b}} / \tau_{\mathrm{b}} \\
& \mathrm{d}\left(\Delta m_{\mathrm{b}}\right) / \mathrm{d} t=-\left(\Delta m_{\mathrm{b}} / T_{2 \mathrm{~b}}\right)-\left(\Delta m_{\mathrm{b}} / \tau_{\mathrm{b}}\right)+\Delta m_{\mathrm{a}} / \tau_{\mathrm{a}}
\end{aligned}
$$

where $\Delta m_{\mathrm{a}}$ and $\Delta m_{\mathrm{b}}$ are deviations from the equilibrium transverse spin magnetization in the sites (a) and (b). The solution of these coupled rate equations is the observable normalized NMR transverse relaxation function $M(t)$ of the bi-exponential form (Hazlewood et al. 1974),

$$
M(t)=P_{\mathrm{a}}^{\prime} \exp \left(-t / T_{2 \mathrm{a}}^{\prime}\right)+P_{\mathrm{b}}^{\prime} \exp \left(-t / T_{2 \mathrm{~b}}\right)
$$

where $P_{\mathrm{a}}^{\prime}$ and $P_{\mathrm{b}}$ are the effective fraction contributions of sites (a) and (b) to the total $M(t)$ signal, with $P_{\mathrm{a}}^{\prime}+P_{\mathrm{b}}{ }_{\mathrm{b}}=1$, and $T^{\prime}{ }_{2 \mathrm{a}}$ and $T^{\prime}{ }_{2 \mathrm{~b}}$ are the effective water proton spin-spin relaxation times of sites (a) and (b).

Thus, in terms of Eq. (3), only three independent parameters are available from the experimental $M(t)$ curves: the effective spin-spin relaxation times $T_{2 \mathrm{a}}^{\prime}$ and $T_{2 \mathrm{~b}}^{\prime}$, and the effective contribution $P^{\prime}$ a of the intracellular water proton population to the total $M(t)$ signal, while $P_{\mathrm{b}}^{\prime}=1-P_{\mathrm{a}}^{\prime}$. These parameters can be determined from the $M(t)$ data with an 
accuracy of $3-4 \%$ anticipated on the basis of analysis of numerous time-independent $M(t)$ curves of control samples. However, there are four unknown parameters, as analytically related to $T^{\prime}{ }_{2 \mathrm{a}}, T_{2 \mathrm{~b}}$ and $P_{\mathrm{a}}^{\prime}$ within the theoretical model of Hazlewood et al. (1974): $P_{\mathrm{a}}$ (the actual fraction of the NMR signal due to intracellular water protons), $\tau_{\mathrm{a}}, T_{2 \mathrm{a}}$ and $T_{2 \mathrm{~b}}$, while $P_{\mathrm{b}}=1-P_{\mathrm{a}}$ and $\tau_{\mathrm{b}}=\left(P_{\mathrm{b}} / P_{\mathrm{a}}\right) \cdot \tau_{\mathrm{a}}$, by detailed balancing. It is therefore necessary to determine one of these four parameters independently, and $T_{2 \mathrm{a}}$ was chosen for this purpose. It was measured separately on tightly packed, non-treated erythrocytes without added $\mathrm{MnCl}_{2}$. A value of $78 \pm 3 \mathrm{~ms}$ was obtained. Also, since the parameter $\tau_{\mathrm{a}}$ represents the mean life time of water molecules in the intracellular space before they are transferred to the extracellular space by diffusion, $\tau_{\mathrm{a}}$ is identified as the time constant $\tau_{\text {exch }}$ introduced before, i.e., $\tau_{\mathrm{a}} \equiv \tau_{\text {exch}}$.

Furthermore, for erythrocyte samples with moderate hematocrit values, i.e., having a $P_{\mathrm{b}}$ value only a few times larger than $P_{\mathrm{a}}$, and when the samples contain high concentrations of $\mathrm{MnCl}_{2}$ in the extracellular solvent sufficient to shorten $T_{2 b}$ enough, one has

$$
T_{2 \mathrm{~b}} \ll \tau_{\mathrm{a}}, \tau_{\mathrm{b}},\left(\tau_{\mathrm{b}}=\left(P_{\mathrm{b}} / P_{\mathrm{a}}\right) \cdot \tau_{\mathrm{a}}\right)
$$

and hence

$$
\left(1 / T_{2 \mathrm{~b}}\right)^{2} » 4 /\left(\tau_{\mathrm{a}} \cdot \tau_{\mathrm{b}}\right)
$$

The exact theoretical expression for $T^{\prime}{ }_{2 \mathrm{a}}$ (Hazlewood et al. 1974) is in such a case simplified to:

$$
1 / T_{2 \mathrm{a}}^{\prime}=1 / T_{2 \mathrm{a}}+1 / \tau_{\text {exch }}
$$

As documented below, conditions (4) and (5) were obeyed well in the experiments on samples with $\sim 20 \%$ hematocrit, $h=0.2$, in buffer solution and doped with $10 \mathrm{mM} \mathrm{MnCl}_{2}$. A value of $T_{2 b} \sim 1 \mathrm{~ms}$ was determined directly on the sample of a buffer solution containing $10 \mathrm{mM} \mathrm{MnCl}_{2}$. At room temperature, $\tau_{\text {exch }}$ values of $\sim 14$ to $\sim 17 \mathrm{~ms}$ are well documented for normal human erythrocytes (see, e.g., Lahajnar et al. 2007 and references therein). And, for the present experiments on samples with $h \approx 0.2, P_{\mathrm{b}} / P_{\mathrm{a}} \approx 5.7$, so that, taking average $\tau_{\text {exch }}=\tau_{\mathrm{a}}=15.5 \mathrm{~ms}, \tau_{\mathrm{b}}=\left(P_{\mathrm{b}} / P_{\mathrm{a}}\right) \cdot \tau_{\mathrm{a}} \approx 88 \mathrm{~ms}$. It is thus evident that the requirements (4) and (5) were obeyed, and the relation (6) is well justified in the present case. The expression (6) will be fundamental in analyzing the observed effector-induced modifications of $T^{\prime}{ }_{2 \mathrm{a}}$.

All NMR experiments were performed at a proton resonance frequency of $100 \mathrm{MHz}$ on a Bruker Biospec System (Bruker, Rheinstetten, Germany), equipped with a superconducting magnet (Oxford Instruments Ltd., England). The radiofrequency $\pi$-pulse separation in the Carr-Purcell-Meiboom-Gill (CPMG) sequence was $\Delta t=0.6 \mathrm{~ms}$ for determining
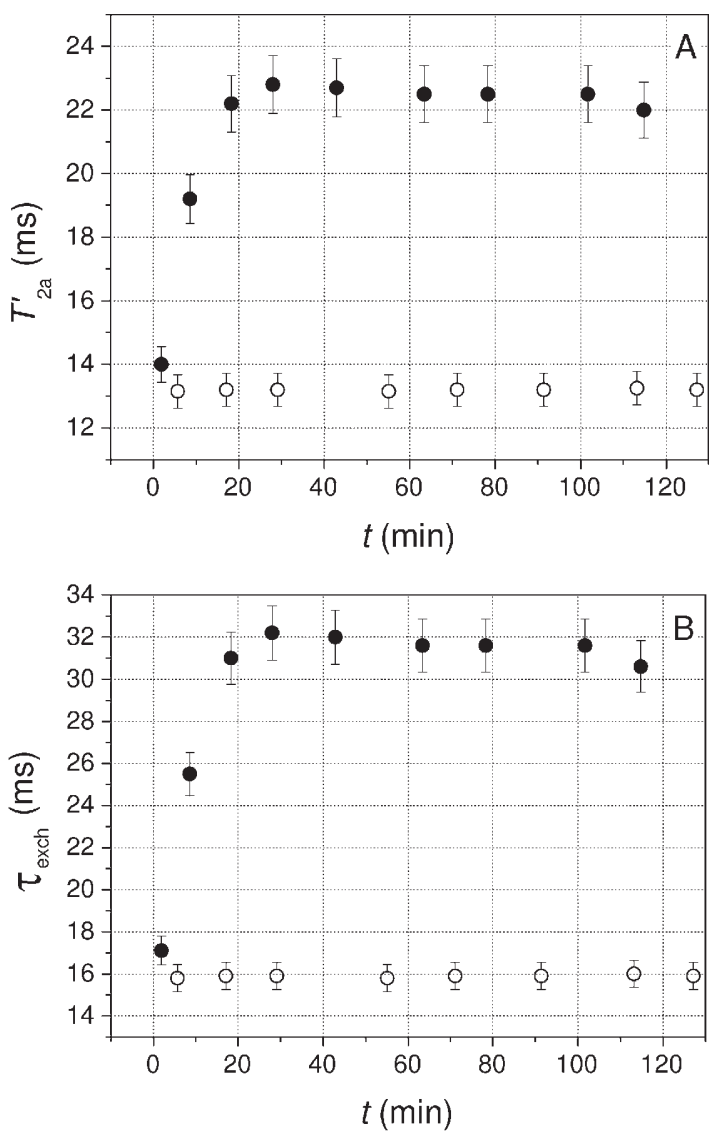

Figure 1. Time variation of the effective intracellular water proton NMR transverse relaxation time $T_{2 \mathrm{a}}(\mathrm{A})$ and of the time parameter $\tau_{\text {exch }}$ for transmembrane diffusional exchange of intracellular water (B) in a $20 \%$ hematocrit sample of human erythrocytes after cell treatment at time $t=0$ with $1 \mathrm{mM}$ PCMB. - sample treated with PCMB; O control.

the relaxation functions $M(t)$, and $6 \mathrm{~ms}$ for determining $T_{2 \mathrm{a}}$. Measurements were made on samples thermostated at $21^{\circ} \mathrm{C}$.

\section{Results and Discussion}

The effect of $1 \mathrm{mM}$ PCMB was determined on the proton NMR transverse relaxation time $T^{\prime}{ }_{2 a}$ and hence on the time constant $\tau_{\text {exch }}$ for transmembrane diffusional exchange of the erythrocyte intracellular water (Fig. 1). $\tau_{\text {exch was }}$ deduced from the $T^{\prime}{ }_{2 a}$ data by means of Eq. (6), where $T_{2 \mathrm{a}} \approx 78 \mathrm{~ms}$. Values of each parameter were found to be increased. Because the erythrocyte intracellular volume, and hence the intracellular water volume, $v$, as well as the cell surface area, $s$, are not influenced by PCMB, the observed extent of $\tau_{\text {exch }}$ increase measures directly the degree of inhibition of the membrane diffusional water 

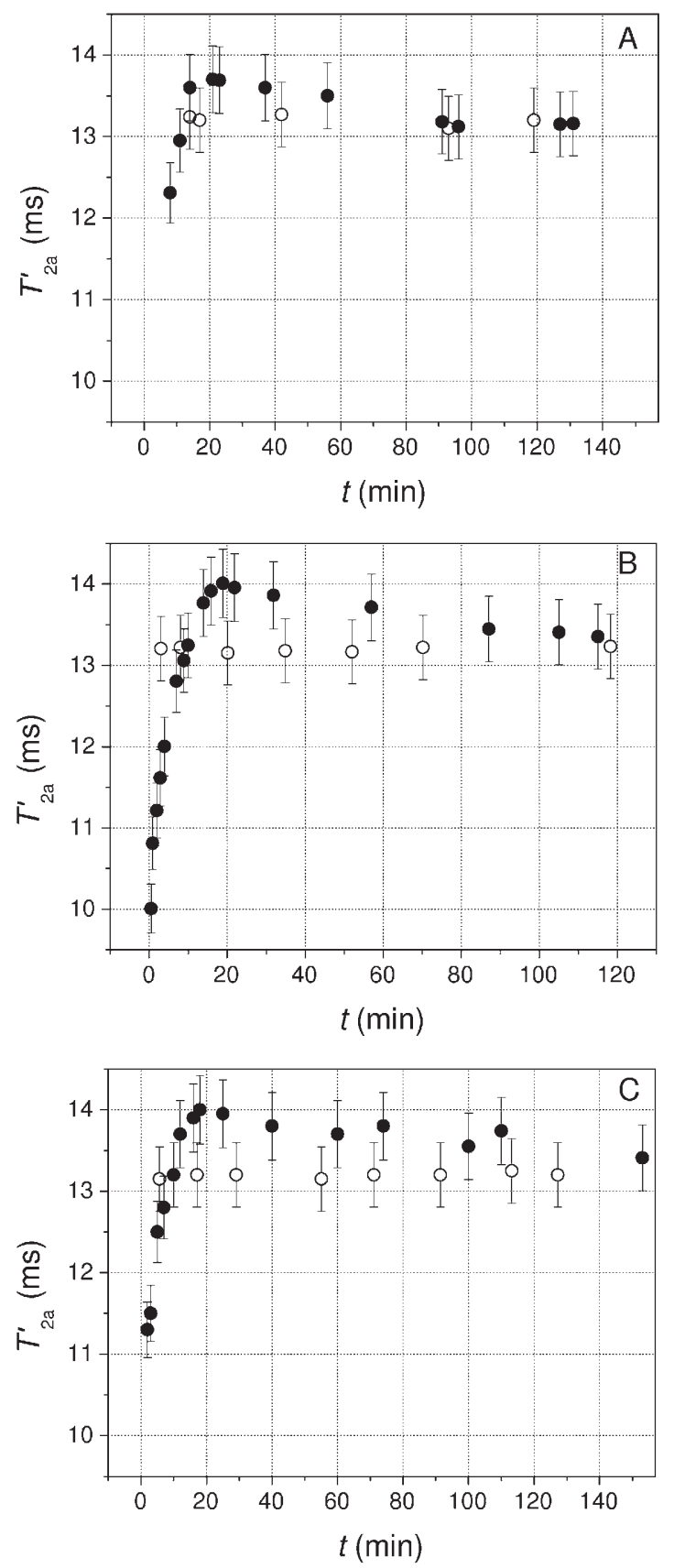

Figure 2. Time variation of the effective intracellular water proton NMR transverse relaxation time $T_{2 \mathrm{a}}{ }_{\mathrm{a}}$ in a $20 \%$ hematocrit sample of human erythrocytes after cell treatment at time $t=0$ with various concentrations of SNP: A. $0.1 \mathrm{mM} \mathrm{SNP}$; B. $0.25 \mathrm{mM}$ SNP; C. $1 \mathrm{mM}$ SNP. sample treated with SNP; O control.

permeability $P_{\mathrm{d}}$, since the two parameters are inversely proportional (Hazlewood et al. 1974; Pirkle et al. 1979; Lahajnar et al. 2007):

$$
P_{\mathrm{d}}=(v / s) \cdot\left(1 / \tau_{\text {exch }}\right)
$$

$\tau_{\text {exch }}$ is increased by a factor 2 . Therefore, according to Eq. (7) the degree of $P_{\mathrm{d}}$ inhibition,

$$
\left(P_{\mathrm{d}}-P_{\mathrm{d}}^{*}\right) / P_{\mathrm{d}}=1-\left(\tau_{\text {exch }} / \tau_{\text {exch }}^{*}\right)
$$

was $\sim 50 \%$, in excellent agreement with the previous result (Finkelstein 1987). In Eq. (8), $P^{\star} \mathrm{d}$ and $\tau^{\star}$ exch refer to modified values of $P_{\mathrm{d}}$ and $\tau_{\text {exch }}$. Thus, since the membrane lipidmediated and AQP1 channel-mediated pathways of water permeation contribute almost equally to the total membrane $P_{\mathrm{d}}$ of human erythrocytes (Finkelstein 1987), the observed degree of $P_{\mathrm{d}}$ inhibition implies that $1 \mathrm{mM}$ PCMB in the $20 \%$ hematocrit sample was sufficient to block all available AQP1 channels.

Fig. 2 shows the time variation of the parameter $T_{2 \mathrm{a}}{ }_{2 \mathrm{a}}$ as a function of SNP concentration. The time course of induced $T^{\prime}$ a variation is characterized by a sharp initial drop to well below the control $T^{\prime}{ }_{2 a}$ level, followed by a steady increase to a maximum, whose value exceeds the control $T_{2 \mathrm{a}}^{\prime}$ value. The initial $T^{\prime}{ }_{2 \mathrm{a}}$ drop is probably the consequence of ${ }^{\bullet} \mathrm{NO}$ released in reduction of SNP by thiol groups of proteins on the erythrocyte (Grossi and D’Angelo 2005). Namely, when some ${ }^{\circ} \mathrm{NO}$ enters into the cell, there it becomes paramagnetic by binding to the heme $\mathrm{Fe}^{2+}$ of hemoglobin which is the most abundant protein in erythrocyte interior (Wajnberg et al. 1996; Cooper 1999). Due to magnetic interactions between electronic spins of heme-bonded paramagnetic -NO and proton spins of neighbouring water molecules, the overall proton relaxation rate $1 / T_{2 a}$ of intracellular water is effectively enhanced to $1 / T^{\star}{ }^{2}$ a which, according to Eq. (6), results in the observed $T_{2 a}^{\prime}$ shortening. Most of released 'NO, due to its lipophilic character, stays in the membrane where it can be oxidized by oxygen to $\mathrm{N}_{2} \mathrm{O}_{3}$ (Zhang et al. 2009). This dinitrogen trioxide can freely diffuse and react with thiol groups to produce an S-nitroso derivative.

Extent of SNP-induced $T^{\prime}{ }_{2 a}$ drop would be expected to increase with increasing amount of administered SNP if in view of proportionality of the paramagnetically enhanced water proton relaxation rate $1 / T^{*} 2 \mathrm{a}$ with the existing transient concentration of heme $\mathrm{Fe}^{2+}$-bound paramagnetic ${ }^{\circ} \mathrm{NO}$ which decreases with time (see below) - it were detected at the same delay after SNP addition and if the number of available hemoglobin $\mathrm{Fe}^{2+}$ iron species exceeds that of released 'NO molecules. The latter condition appears to be fulfilled, as with 250 millions of hemoglobin ( $\mathrm{Hb}$ ) molecules in a single erythrocyte and with $\sim 6.5 \times 10^{8}$ erythrocytes in a $0.3 \mathrm{ml}$ sample of $20 \%$ hematocrit there are $\sim 1.6 \times 10^{17} \mathrm{Hb}$ molecules (and hence twice as many heme $\mathrm{Fe}^{2+}$ atoms in the case of $50 \%$ saturation with oxygen), while for $1 \mathrm{mM} \mathrm{SNP} \mathrm{a}$ similar number of $\sim 1.8 \times 10^{17}$ NO molecules are released, but of which only a part may be considered to react with heme $\mathrm{Fe}^{2+}$. On the other hand, the former condition was not satisfied because of different experimentally achieved delays 
in starting $T^{\prime}{ }_{2 \mathrm{a}}$ drop determination for each of the examined SNP concentrations. As a consequence, for instance, the largest $T^{\prime}{ }_{2 \mathrm{a}}$ drop to $10.0 \mathrm{~ms}$ was observed for $0.25 \mathrm{mM} \mathrm{SNP}$ and a delay of $30 \mathrm{~s}$ (Fig. 2B), while a smaller one to $11.3 \mathrm{~ms}$ for $1 \mathrm{mM} \mathrm{SNP}$ and a larger delay of $2 \mathrm{~min}$ (Fig. 2C). It is worth to repeat that the largest SNP-induced initial $T_{2 \mathrm{a}}^{\prime}$ drop was observed for the shortest experimentally accessible delay of $30 \mathrm{~s}$ in starting $T_{2 \mathrm{a}}$, determination after SNP addition to the sample (Fig. 2B). This minimum delay in the present study was due to the time needed to add SNP to the sample, to place the sample into the NMR radiofrequency coil, and the time for the NMR signal acquisition.

The fact that $T_{2 \mathrm{a}}$, after the sharp initial drop, increases with time is indicative of the decreasing intracellular concentration of the paramagnetic species after sample treatment with SNP. We assume, according to (Lahajnar et al. 2007), that ${ }^{\circ} \mathrm{NO}$ is bound to the heme $\mathrm{Fe}^{2+}$. This is in line with the well known proportionality relationship between a paramagnetically enhanced water proton NMR relaxation rate $1 / T_{2}$ and concentration of dissolved paramagnetic centres (Abragam 1961). In the present case this relation refers to a paramagnetically enhanced proton relaxation rate contribution $1 / T^{*}{ }_{2 \mathrm{a}}$ to the total $1 / T^{\prime}{ }_{2 \mathrm{a}}$ of intracellular water (see Eq. (6)) and the concentration of the existing intracellular paramagnetic ${ }^{\circ} \mathrm{NO}$ population. The already mentioned oxidation of ${ }^{\circ} \mathrm{NO}$ in the cell interior accounts for the disappearance of the ${ }^{\bullet} \mathrm{NO}$. Therefore, after an initial drop $T_{2 \mathrm{a}}^{\prime}$ increases with time towards the control $T_{2 \mathrm{a}}^{\prime}$ level. Accordingly, the pool of paramagnetic ${ }^{\circ} \mathrm{NO}$ appears to be entirely exhausted when $T^{\prime}{ }_{2 \mathrm{a}}$ exceeds the control $T^{\prime}{ }_{2 \mathrm{a}}$ level and attains a maximum some $20 \mathrm{~min}$ after SNP addition to the samples (Fig. 2). Obviously, at this stage it is expected that $T^{*}{ }_{2 \mathrm{a}} \equiv T_{2 \mathrm{a}}=78 \mathrm{~ms}$. However, since $T_{2 \mathrm{a}}^{\prime}$ is observed to lengthen over its control level (see Fig. 2), and neither the intracellular water volume $v$ nor the cell surface area $s$ are affected by SNP (Lahajnar et al. 2007), it follows from Eq. (6) that it is the exchange time $\tau_{\text {exch }}$ which has been lengthened (to $\tau_{\text {exch }}^{\star}$ ) by this stage. Alternatively, according to Eq. (7), the erythrocyte membrane water permeability $P_{\mathrm{d}}$ has been somewhat suppressed, probably by the reaction of $\mathrm{N}_{2} \mathrm{O}_{3}$ with the critical thiol group of AQP1 (Zhang et al. 2009).

Similar extents of the temporary deviation of $T_{2 a}^{\prime}$ relative to the control $T_{2 \mathrm{a}}$ level, observed $\sim 20 \mathrm{~min}$ after SNP administration to erythrocyte samples for the examined SNP concentrations (Fig. 2), may be considered as due to a largely prevailing number of potentially available ${ }^{\circ} \mathrm{NO}$ molecules relative to that of AQP1 critical thiol groups in the samples. Namely, even the lowest examined $0.1 \mathrm{mM}$ SNP can generate $\sim 1.8 \times 10^{16} \mathrm{NO}$ molecules in a $0.3 \mathrm{ml}$ sample, while with $\sim 6.5 \times 10^{8}$ erythrocytes in that $20 \%$ hematocrit sample and with $\sim 2 \times 10^{5}$ AQP1 monomers (and hence the same number of critical SH-groups) per erythrocyte, there are $\sim 1.3 \times 10^{14}$ critical SH-groups in the sample. Hence, similar extents of the $T_{2 \mathrm{a}}^{\prime}$ deviation above the control $T^{\prime}{ }_{2 \mathrm{a}}$ level, observed for the examined SNP concentrations, may be considered to indicate that there is sufficient released ${ }^{\circ} \mathrm{NO}$ (or $\mathrm{N}_{2} \mathrm{O}_{3}$ as one of its oxidation products) to interact stepwise with available Cys189 residues in the samples.

The quantitative aspects of the observed PCMB-induced suppression of the erythrocyte membrane diffusional water permeability as well as of the observed SNP-induced time variation of $T_{2 \mathrm{a}}^{\prime}$ and of concomitant modifications of $T_{2 \mathrm{a}}$ and $\tau_{\text {exch }}$ are considered below.

Treatment of $20 \%$ hematocrit suspensions of human erythrocytes with $1 \mathrm{mM}$ PCMB results in a $50 \%$ inhibition of the total membrane diffusion water permeability $P_{\mathrm{d}}$. As the membrane lipid and AQP1 channel pathways of water permeation across human erythrocyte membrane contribute almost equally to the total membrane $P_{\mathrm{d}}$ (Finkelstein 1987), it follows that $1 \mathrm{mM}$ PCMB is sufficient to block all available AQP1 water channels in these suspensions.

Nitric oxide, $\mathrm{NO}$, because of its hydrophobic nature, readily concentrates in and diffuses across cell membranes, and has a relatively short life time of several seconds in biosystems due to oxidation with dissolved molecular oxygen, either in the membrane or the cell interior (Kharitonov et al. 1994; Möller et al. 2007). The partition coefficients of $\mathrm{O}_{2}$ and ${ }^{\circ} \mathrm{NO}$ between lipophilic membrane and water are 5 and 9, respectively (Möller et al. 2007). Additionally, the presence of red blood cells poses another factor contributing to the life time of free ${ }^{\bullet} \mathrm{NO}$, due to the high affinity of hemoglobin for ${ }^{\circ} \mathrm{NO}$ and its high concentration (Eich et al. 1996).

The observed SNP-induced time variation of $T^{\prime}{ }_{2 a}$ (Fig. 2) is discussed below to be due to the effects of released ${ }^{\circ} \mathrm{NO}$, both on the intrinsic proton NMR transverse relaxation time $T_{2 \mathrm{a}}$ of intracellular water and on the time constant $\tau_{\text {exch }}$ for transmembrane diffusional exchange of intracellular water. The latter change in turn may directly involve a modification of the erythrocyte membrane diffusional water permeability $P_{\mathrm{d}}$, as in the case of the sample treated with SNP, $\tau_{\text {exch }}$ and $P_{\mathrm{d}}$ remain inversely proportional, i.e., $P_{\mathrm{d}}=(v / s) \cdot\left(1 / \tau_{\text {exch }}\right)$ by Eq. (7), because neither the intracellular water volume $v$ of a single erythrocyte (about 0.70 of its total isotonic volume) nor the erythrocyte membrane surface area $s$ are influenced by SNP treatment (Lahajnar et al. 2007). The room temperature ratio $v / s$ for the isotonic human erythrocyte is $4.56 \times 10^{-5} \mathrm{~cm}$ (Brahm 1982). As already noted, the SNP-induced modification of intrinsic $T_{2 a}$, implied by the initial rapid drop of $T_{2 a}^{\prime}$ (Fig. 2), is considered to be due to an enhanced intracellular water proton NMR transverse relaxation rate $1 / T^{\star} 2 \mathrm{a}$. This arises from magnetic interactions between the induced electronic magnetic moments in those -NO molecules, which entered the erythrocyte intracellular space and noncovalently bonded to the heme $\mathrm{Fe}^{2+}$ atoms of deoxyhemoglobin and the proton spins of neighbouring water molecules in the distal part of the heme pocket. By 
'NO binding to the sixth position of the heme iron, the $\mathrm{Fe}$ ion remains ferrous $\left(\mathrm{Fe}^{2+}\right.$ with electronic spin $S=0$ ), while noncovalently bonded ${ }^{\circ} \mathrm{NO}$ becomes paramagnetic, since it acquires the electronic spin $S=1 / 2$ (Eich et al. 1996; Wajnberg et al. 1996; Cooper 1999). Due to this magnetic interaction the otherwise rather slow intrinsic proton relaxation rate $1 / T_{2 \mathrm{a}}$ (with $T_{2 \mathrm{a}} \approx 78 \mathrm{~ms}$ ) of water molecules in the heme pocket becomes greatly enhanced. Consequently, the fast exchange of these $\mathrm{H}_{2} \mathrm{O}$ molecules with the rest of the water in the cell interior results in an overall paramagnetically enhanced proton relaxation rate $1 / T^{*} 2 \mathrm{a}$, which is proportional to the existing transient concentration of heme-bound paramagnetic ${ }^{\circ} \mathrm{NO}$. (For NMR relaxation enhancement effects by paramagnetic centres, see, e.g., Abragam 1961). It should be noted that the paramagnetism of ${ }^{\bullet} \mathrm{NO}$ molecules is not observed unless their ground state $\pi$-orbital degeneracy is lifted by interaction of ${ }^{\circ} \mathrm{NO}$ with the electrostatic field of, for example, bound metal ions (as in the case of noncovalent ${ }^{\circ} \mathrm{NO}$ binding to the ferrous heme iron atom of hemoglobin). The half-life time for the $\mathrm{Hb}$ $\mathrm{Fe}^{2+}{ }^{-} \mathrm{NO}$ complex is $\sim 15 \mathrm{~min}$ in the absence of the ${ }^{\bullet} \mathrm{NO}$ source (Cooper 1999). This time appears to correlate with the present observation that the effective relaxation time $T^{\prime}{ }_{2 \mathrm{a}}$ attained a maximum value some $20 \mathrm{~min}$ after SNP was added to the erythrocyte suspension (see Fig. 2), when at this stage the pool of intracellular bound paramagnetic ${ }^{\circ} \mathrm{NO}$ is exhausted (i.e., $T^{*} 2 \mathrm{a} \equiv T_{2 \mathrm{a}}$ ). Because the maximum $T_{2 \mathrm{a}}{ }_{2 \mathrm{a}}$ value lies above the control $T_{2 \mathrm{a}}^{\prime}$ level and, since $T^{*}{ }_{2 \mathrm{a}}=T_{2 \mathrm{a}}$, it follows from Eq. (6) that $T^{\prime}{ }_{2 a}$ is increased because of an SNP-induced increase of $\tau_{\text {exch }}$ to $\tau^{\star}$ exch. However, as the data of Fig. 2 suggest, a relatively small part of this $\tau_{\text {exch }}$ modification can be ignored when compared to that of the initial $T_{2 \mathrm{a}}$ paramagnetic shortening, so according to Eq. (6) the early $T_{2 \mathrm{a}}$, data may be approximated as

$$
1 / T_{2 \mathrm{a}}^{\prime} \approx 1 / T_{2 \mathrm{a}}^{*}+1 / \tau_{\mathrm{exch}}
$$

In view of Eq. (9), the initial time course of $T_{2 \mathrm{a}}{ }^{\mathrm{a}}$ (particularly that given in Fig. 2B) may be taken to reflect the kinetics of the paramagnetic ${ }^{\circ} \mathrm{NO}$ concentration decrease in the earlier stage following the addition of SNP to the erythrocyte sample.

As noted earlier, the parameter $\tau_{\text {exch }}$ appears to be modified by SNP, i.e., is lengthened to $\tau^{\star}$ exch, because of the reduced transmembrane water exchange that is suggested to result from partial steric hindrance of water flow through membrane AQP1 channels caused by ${ }^{\circ} \mathrm{NO}$ bound to the SH-groups of channel Cys189 residues (Lahajnar et al. 2007). Hence the relaxation time $T_{2 a}^{\prime}$ is, according to Eq. (6), contributed to by both modified parameters $T^{*}$ a and $\tau^{*}$ exch as

$$
1 / T_{2 \mathrm{a}}^{\prime}=1 / T_{2 \mathrm{a}}^{*}+1 / \tau_{\text {exch }}^{*}
$$

Therefore, when $T_{2 \mathrm{a}}{ }_{\mathrm{a}}$ of the SNP-treated erythrocyte sample returned to the control value of $13.2 \mathrm{~ms}$ (Fig. 2), the sum of existing modified rates $1 / T^{*}{ }_{2 a}$ and $1 / \tau^{*}$ exch matches that of the corresponding control rates $1 / T_{2 \mathrm{a}}$ and $1 / \tau_{\text {exch }}$, i.e.,

$$
1 / T_{2 \mathrm{a}}^{\prime} \equiv 1 / T_{2 \mathrm{a}}^{*}+1 / \tau_{\text {exch }}^{*}=1 / T_{2 \mathrm{a}}+1 / \tau_{\text {exch }}
$$

Thus one can conclude that, at this point, a paramagnetically-induced relaxation increment in $1 / T_{2 a}$, due to the still existing intracellular paramagnetic heme bound ${ }^{\circ} \mathrm{NO}$, is compensated by a decrement in $1 / \tau_{\text {exch }}$ because of proposed steric slowing down of transmembrane water exchange by bound ${ }^{\circ} \mathrm{NO}$ on AQP1 channel Cys189 residues.

Further inspection of the data in Fig. 2 shows that $\sim 20 \mathrm{~min}$ after SNP addition to the sample the parameter $T^{\prime}{ }_{2 \mathrm{a}}$ attained a maximum value of $14.0 \mathrm{~ms}$ for the $0.25 \mathrm{mM}$ and $1 \mathrm{mM}$ SNP concentrations (Fig. 2B and C), and somewhat less for that of $0.1 \mathrm{mM}$ SNP (Fig. 2A). It then slowly approached the control $T_{2 \mathrm{a}}{ }_{2 \mathrm{a}}$ value. It can be concluded that, at the stage of the $T_{2 \mathrm{a}}$ maximum, intracellular heme-bound paramagnetic 'NO was entirely depleted so that $T^{*}{ }_{2 \mathrm{a}}=T_{2 \mathrm{a}}$ (with $T_{2 \mathrm{a}}$ equal to the intrinsic value of $78 \mathrm{~ms}$ ), and only the exchange time constant remained modified (lengthened) to $\tau^{\star}$ exch due to bound ${ }^{\circ} \mathrm{NO}$ on AQP1 Cys189 residues. Therefore, at this stage, from Eq. (10),

$$
1 / T_{2 \mathrm{a}}^{\prime}=1 / T_{2 \mathrm{a}}+1 / \tau_{\text {exch }}^{*}
$$

The initial relatively steep time course of $T_{2 \mathrm{a}}{ }_{2 \mathrm{a}}$ increase thus appears to be correlated with the life time of bound paramagnetic ${ }^{\circ} \mathrm{NO}$ on the heme $\mathrm{Fe}^{2+}$ iron (Cooper 1999), and the subsequent decrease of $T_{2 \mathrm{a}}$ (toward the control $T_{2 \mathrm{a}}{ }_{2 \mathrm{a}}$ ) suggests a much longer but finite life time of Cys189 nitrosothiols in the membrane AQP1 channels. The latter is in line with the report (Grossi and D'Angelo 2005) that, when SNP reacts with a thiol-containing species, the released NO forms labile R-S-NO products. These can in turn serve as a storage and transporter of ${ }^{\bullet} \mathrm{NO}$ in vivo.

The degree of proposed SNP-induced suppression of the erythrocyte membrane diffusional water permeability $P_{\mathrm{d}}$ can be estimated from Eq. (7). This is possible because in erythrocyte treatment with SNP, $\tau_{\text {exch }}$ for the transmembrane exchange of intracellular water is modified (i.e., lengthened to $\tau^{\star}$ exch as shown before), while the cell volume (and hence the intracellular water volume $v$ ) and the cell membrane surface $s$ are unchanged (Lahajnar et al. 2007). The SNPinduced lengthening of $\tau_{\text {exch }}$ to $\tau^{\star}$ exch was suggested previously (Lahajnar et al. 2007) as being due to steric restriction of water permeation through membrane AQP1 channels caused by bound ${ }^{\circ} \mathrm{NO}$ on Cys189 residues in the channel pores. Then, if $\tau_{\text {exch }}$ is lengthened to $\tau_{\text {exch }}^{*}$, the degree of $P_{\mathrm{d}}$ suppression, $\left(P_{\mathrm{d}}-P^{*}\right) / P_{\mathrm{d}}$, is calculated by means of expression (8). The unmodified $\tau_{\text {exch }}$ value of $15.9 \mathrm{~ms}$ is de- 
termined by means of Eq. (6), using the control value $T_{2 \mathrm{a}}^{\prime}=$ $13.2 \mathrm{~ms}$ (Fig. 2) and the intrinsic value $T_{2 \mathrm{a}}=78 \mathrm{~ms}$. With $v / s=4.56 \times 10^{-5} \mathrm{~cm}$ (Brahm 1982), the unmodified $P_{\mathrm{d}}$ value calculated by means of Eq. (7) is $P_{\mathrm{d}}=2.9 \times 10^{-3} \mathrm{~cm} / \mathrm{s}$. This is well within the reported range of $P_{\mathrm{d}}$ values for the human erythrocyte membrane (Brahm 1982; Finkelstein 1987). The

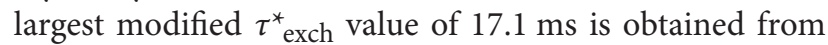
Eq. (12), inserting $T_{2 \mathrm{a}}=78 \mathrm{~ms}$ as before and the value $T_{2 \mathrm{a}}{ }_{2 \mathrm{a}}$ of $14.0 \mathrm{~ms}$ taken at the maxima of Figs. $2 \mathrm{~B}$ and $2 \mathrm{C}$. Thus the degree of $P_{\mathrm{d}}$ suppression reached with $1 \mathrm{mM}$ and $0.25 \mathrm{mM}$ SNP is calculated from Eq. (8) as 7.0\%, somewhat less than reported earlier (Lahajnar et al. 2007). The same extent of $P_{\mathrm{d}}$ suppression (7.0\%) obtained with $1 \mathrm{mM}$ and $0.25 \mathrm{mM} \mathrm{SNP}$ would imply that in both cases there was sufficient ${ }^{\circ} \mathrm{NO}$ to reach and react directly with Cys 189 residues in the $20 \%$ hematocrit sample or transform first to $\mathrm{N}_{2} \mathrm{O}_{3}$ and then react with the thiol group in the AQP1 channel.

Such a suppression of water permeability through AQP1 may be understood in a more general and physiological frame as follows. ${ }^{\circ} \mathrm{NO}$ has a high affinity for $\mathrm{Fe}^{2+}$ ions and their interaction is spontaneous. Thus intracellular ${ }^{\bullet} \mathrm{NO}$ will occupy available and accessible positions around $\mathrm{Fe}^{2+}$ ions if alternative modes of ${ }^{\bullet} \mathrm{NO}$ transformation are saturated or less competitive. $\mathrm{NO}$ can also react as a free radical with any accessible thiol group to give RS-NO, either directly, via $\mathrm{N}_{2} \mathrm{O}_{3}$ as a product of ${ }^{\bullet} \mathrm{NO}$ oxidation, or by reaction with ${ }^{+}$NO. S-nitrosation is also a spontaneous reaction.

In inflammation, the local production of ${ }^{\bullet} \mathrm{NO}$ in tissue is high, increasing the number of its spontaneous reactions, including those with Cys189 thiol group of AQP1 as suppressing erythrocyte membrane water permeability. The most important biological effect of ${ }^{\bullet} \mathrm{NO}$ is vasodilation, possibly the consequence of activation of guanylate cyclase by ${ }^{\circ} \mathrm{NO}$ binding to its $\mathrm{Fe}^{2+}$. S-nitrosation of Cys189 (AQP1) would ultimately result in slowing down erythrocyte volume changes, while the reaction with guanylate cyclase, resulting in vasodilation, may compensate the former effect.

-NO production is under the control of endothelial and neuronal NO-synthases under normal physiological conditions. In the case of inflammation, however, expression of NO-synthase is induced, producing surplus ${ }^{\circ} \mathrm{NO}$. In this view, the reduced water permeability in erythrocytes may be compensated by concomitant vasodilation that is also achieved with other mediators of inflammation, like histamine. The observed effect of SNP on erythrocyte water permeability appears to fit well into a more general frame of physiological functions of ${ }^{\bullet} \mathrm{NO}$ and its products.

\section{Conclusion}

The time-course of the intracellular water proton apparent relaxation time $T_{2 \mathrm{a}}{ }^{\mathrm{a}}$ in human erythrocyte suspensions treated with SNP ( ${ }^{\circ} \mathrm{NO}$ donor) has been proposed to be due to the two mechanisms by which released ${ }^{\circ} \mathrm{NO}$ affects the relaxation time $T_{2 \mathrm{a}}{ }_{2 \mathrm{a}}$ in these samples. The two mechanisms refer, respectively, to increase in the intracellular water proton intrinsic relaxation rate $1 / T_{2 \mathrm{a}}$ due to paramagnetism of ${ }^{\bullet} \mathrm{NO}$ bound to ferrous iron atoms of intracellular hemoglobin, and decrease of the diffusional water permeability $P_{\mathrm{d}}$ of erythrocyte membrane by partial temporary inhibition of AQP1 channels - either by direct reaction of the channel Cys189 residue with ${ }^{\bullet} \mathrm{NO}$ or with one of the ${ }^{\bullet} \mathrm{NO}$ oxidation products such as $\mathrm{N}_{2} \mathrm{O}_{3}$.

As far as the former mechanism is concerned, it refers to the observed initial sharp $T^{\prime}{ }_{2 \mathrm{a}}$ drop that is suggested to arise from the enhancement of the intrinsic intracellular water proton relaxation rate $1 / T_{2 \mathrm{a}}$ by paramagnetism of that fraction of ${ }^{\bullet} \mathrm{NO}$, which has entered the cell interior and noncovalently bonded to ferrous iron atoms of deoxyhemoglobin. The subsequent steady return of $T_{2 \mathrm{a}}{ }_{2 \mathrm{a}}$ towards the control level is indicative of the decreasing concentration of $\mathrm{Fe}^{2+}$-bonded paramagnetic ${ }^{\circ} \mathrm{NO}$ in deoxyhemoglobin. This conclusion stems from the proportionality between the enhanced intracellular water proton relaxation rate $1 / T^{*}$ a (that in terms of Eq. (9) contributes most to the time variation of $1 / T^{\prime}{ }_{2 a}$, at least in the early stage after sample treatment with SNP) and the existing concentration of the intracellular paramagnetic ${ }^{\circ} \mathrm{NO}$ population, the latter being decreased by dissociation of the ${ }^{\bullet} \mathrm{NO}$ paramagnetic complex with iron in hemoglobin, followed by ${ }^{\bullet} \mathrm{NO}$ oxidation.

Then, when $T_{2 \mathrm{a}}$, exceeded its control level and reached a maximum, the pool of paramagnetic ${ }^{\circ} \mathrm{NO}$ was exhausted. Despite the fact that the maximum $T_{2 \mathrm{a}}{ }_{2 \mathrm{a}}$ deviation is relatively small, its magnitude nevertheless evidently exceeds the 3-4\% errors of $T_{2 \mathrm{a}}^{\prime}$ data. Accordingly, the observed $T_{2 \mathrm{a}}{ }_{2 \mathrm{a}}$ deviation above the control level is supposed to be related to a SNP-induced lengthening of the time parameter $\tau_{\text {exch }}$ for transmembrane diffusional exchange of intracellular water and hence to temporary suppression of the erythrocyte membrane diffusional water permeability $P_{\mathrm{d}}$. This may be considered to result from steric restriction to water permeation through membrane AQP1 channels posed by bound -NO on critical Cys189 residues located in the channel pores. Equal degrees of total $P_{\mathrm{d}}$ suppression by $7.0 \%$ are deduced for $1 \mathrm{mM}$ and $0.25 \mathrm{mM}$ SNP, suggesting that there is sufficient released ${ }^{\circ} \mathrm{NO}$ to interact stepwise with all available Cys189 residues in the samples.

Treatment of a $20 \%$ hematocrit sample of human erythrocytes with $1 \mathrm{mM}$ PCMB resulted in a 50\% inhibition of the total membrane $P_{\mathrm{d}}$, i.e., in full inhibition of the well documented AQP1 channel water permeability. It is therefore concluded that $\mathrm{SNP}$, via released ${ }^{\circ} \mathrm{NO}$, can react with the $\mathrm{SH}$ groups (as does PCMB) of the Cys 189 residues located near the constriction of the erythrocyte membrane AQP1 channels and form labile RS-NO products, with the bound ${ }^{\circ} \mathrm{NO}$ 
imposing a less effective barrier to water permeation than the larger carboxyphenylmercuryl residue from PCMB.

Acknowledgement. This work was financially supported by the Slovenian Research Agency within the programs P1-0125 and P10208. The authors thank Professor Roger Pain for critical reading of the manuscript.

\section{References}

Abragam A. (1961): The Principles of Nuclear Magnetism. Oxford Univ. Press (Clarendon). London and New York

Brahm J. (1982): Diffusional water permeability of human erythrocytes and their ghosts. J. Gen. Physiol. 79, 791-819; doi:10.1085/jgp.79.5.791

Cooper C. E. (1999): Nitric oxide and iron proteins. Biochim. Biophys. Acta 1141, 290-309

Denker B. M., Smith B. L., Kuhajda F. P., Agre P. (1988): Identification, purification, and partial characterization of a novel Mr 28,000 integral membrane protein from erythrocytes and renal tubules. J. Biol. Chem. 263, 15634-15642

Eich R. F., Li T., Lemon D. D., Doherty D. H., Curry S. R., Aitken J. F., Mathews A. J., Johnson K. A., Smith R. D., Phillips G. N. Jr., Olson J. S. (1996): Mechanism of NO-induced oxidation of myoglobin and hemoglobin. Biochemistry 35, 6976-6983; doi:10.1021/bi960442g

Finkelstein A. (1987): Water Movement through Lipid Bilayers, Pores, and Plasma Membranes: Theory and Reality. Wiley, New York

Grossi L., D’Angelo S. D. (2005): Sodium nitroprusside mechanism of NO release mediated by sulfhydryl-containing molecules. J. Med. Chem. 48, 2622-2626; doi:10.1021/ jm049857n

Hazlewood C. F., Chang D. C., Nichols B. L., Woessner D. E. (1974): Nuclear magnetic resonance transverse relaxation times of water protons in skeletal muscle. Biophys. J. 14, 583-606; doi:10.1016/S0006-3495(74)85937-0

Herold S., Röck G. (2005): Mechanistic studies of S-nitrosothiol formation by $\mathrm{NO} / \mathrm{O} 2$ and by $\mathrm{NO} /$ methemoglobin. Arch. Biochem. Biophys. 436, 386-396; doi:10.1016/ j.abb.2005.02.013

Huang Z., Louderback J. G., Goyal M., Azizi F., King S. B., KimShapiro D. B. (2001): Nitric oxide binding to oxygenated hemoglobin under physiological conditions. Biochim. Biophys. Acta 1568, 252-260

Huang Z., Ucer K. B., Murphy T., Williams R. T., King S. B., KimShapiro D. B. (2002): Kinetics of nitric oxide binding to R-state hemoglobin. Biochem. Biophys. Res. Commun. 292, 812-818; doi:10.1006/bbrc.2002.6730

Ignarro L. J. (2000a): Nitric Oxide: Biology and Pathobiology. Academic Press, San Diego

Ignarro L. J. (2000b): Nitric oxide as a unique signalling molecule in the vascular system: a historical review. J. Physiol. Pharmacol. 53, 503-514

Kharitonov V. G., Sundquist A. R, Sharma V. S. (1994): Kinetics of nitric oxide autoxidation in aqueous solution. J. Biol. Chem. 269, 5881-5883
Lahajnar G. (1992): Diffusional water permeability of bovine erythrocytes: a pulse nuclear magnetic resonance study. Croat. Chem. Acta 65, 191-200

Lahajnar G., Pečar S., Sepe A. (2007): Na-nitroprusside and $\mathrm{HgCl} 2$ modify the water permeability and volume of human erythrocytes. Bioelectrochemistry 70, 462-468; doi:10.1016/j.bioelechem.2006.07.009

Liao J. C., Hein T. W., Vaughn M. W., Huang K. T., Kuo L. (1999): Intravascular flow decreases erythrocyte consumption of nitric oxide. Proc. Natl. Acad. Sci. USA 96, 8757-8761; doi:10.1073/pnas.96.15.8757

Macey R. I., Farmer R. E. I. (1970): Inhibition of water and solute permeability in human red cells. Biochim. Biophys. Acta 211, 104-106; doi:10.1016/0005-2736(70)90130-6

Möller M. N., Li Q., Vitturi D. A., Robinson J. M., Lancaster J. R. Jr., Denicola A. (2007): Membrane "lens" effect: Focusing the formation of reactive nitrogen oxides from the 'NO/O2 reaction. Chem. Res. Toxicol. 20, 709-714; doi:10.1021/tx700010h

Pirkle J. L., Ashley D. L., Goldstein J. H. (1979): Pulse nuclear magnetic resonance measurements of water exchange across the erythrocyte membrane employing a low Mn concentration. Biophys. J. 25, 389-406; doi:10.1016/ S0006-3495(79)85311-4

Preston G. M., Agre P. (1991): Isolation of the cDNA for erythrocyte integral membrane protein of 28 kilodaltons: member of an ancient channel family. Proc. Natl. Acad. Sci. USA 88, 11110-11114; doi:10.1073/pnas.88.24.11110

Preston G. M., Carrol T. P., Guggino W. B., Agre P. (1992): Appearance of water channels in Xenopus oocytes expressing red cell CHIP28 protein. Science 256, 385-387; doi:10.1126/ science.256.5055.385

Preston G. M., Jung J. S., Guggino W. B., Agre P. (1993): The mercury-sensitive residue at cysteine 189 in the CHIP28 water channel. J. Biol. Chem. 268, 17-20

Shaw A. W., Vosper A. J. (1977): Solubility of nitric oxide in aqueous and nonaqueous solvents. J. Chem. Soc. Faraday Trans. 1. 73, 1239-1244; doi:10.1039/f19777301239

Snyder S. H., Bredt D. S. (1992): Biological roles of nitric oxide. Sci. Am. 266, 74-77; doi:10.1038/scientificamerican0592-68

Subczynski W. K., Hyde J. S. (1983): Concentration of oxygen in lipid bilayers using a spin-label method. Biophys. J. 41, 283-286; doi:10.1016/S0006-3495(83)84439-7

Ye J. S., Ottova A., Tien H. T., Sheu F. S. (2001): Nitric oxide enhances the capacitance of self-assembled, supported bilayer lipid membranes. Electrochemistry Communications 3, 580-584; doi:10.1016/S1388-2481(01)00227-2

Wajnberg E., Bemski G., El-Jaick L. J., Alves O. C. (1996): Nitrosyl hemoglobins: EPR above 80 K. Int. J. Biol. Macromol. 18, 231-235; doi:10.1016/0141-8130(95)01078-5

Zhang H., Andrekopoulos C., Xu Y., Joseph J., Hogg N., Feix J., Kalyanaraman B. (2009): Decreased S-nitrosation of peptide thiols in the membrane interior. Free Radic. Biol. Med. 47, 962-968; doi:10.1016/j.freeradbiomed.2009.06.031

Received: May 5, 2010

Final version accepted: September 2, 2010 